\title{
Conversion of Glucose and Gluconate to Ethanol in Mineral Salts Medium using Recombinant Escherichia coli Strains
}

\author{
Zhiliang Fan ${ }^{1 *}$, Yaqin Sun ${ }^{1,2}$, Hui Lin ${ }^{1}$, Di Ma ${ }^{1}$ and Shuangyan Han $^{1,3}$ \\ ${ }^{1}$ Department of Biological and Agriculture Engineering, University of California, One Shields Avenue, CA, USA \\ ${ }^{2}$ School of Life Science and Biotechnology, Dalian University of Technology, Dalian, P. R. China \\ ${ }^{3}$ Guangdong Key Laboratory of Fermentation and Enzyme Engineering, School of Bioscience and Bioengineering, South China University of Technology, Guangzhou, P. \\ R. China
}

"Corresponding author: Zhiliang Fan, Department of Biological and Agriculture Engineering, University of California, One Shields Avenue, CA, USA, Tel: 530-754-0317; Fax: 530-752-2640; E-mail: jzfan@ucdavis.edu

Received date: October 05, 2016, Accepted date: October 26, 2016, Published date: November 01, 2016

Copyright: () 2016 Fan Z, et al. This is an open-access article distributed under the terms of the Creative Commons Attribution License, which permits unrestricted use, distribution, and reproduction in any medium, provided the original author and source are credited.

\begin{abstract}
Escherichia coli $\mathrm{AH} 003$, a derivative of E. coli $\mathrm{KO} 11$ with the L-lactate dehydrogenase (Idh) and pyruvate formate lyase ( $p f l)$ genes deleted and its parent strain E. coli KO11 were used as the ethanologen to convert glucose and gluconate to ethanol in M9 minimal medium. E. coli AH003 grew very poorly on glucose in M9 medium. However it achieved rapid growth when gluconate was used as the carbon source. The addition of gluconate to medium containing glucose improved the rate of glucose utilization. In contrast, $E$. coli KO11 grew well on both glucose and gluconate in M9 medium. The addition of gluconate to medium containing glucose did not improve the rate of glucose utilization. We believe that the deletion of the pfl gene in E. coli AH0O3 led to the different fermentation results. The co-fermentation of gluconate and glucose could be a useful strategy to improve the rate of glucose fermentation and decrease nutrient requirements for engineered strains lacking the pfl gene and grown under anaerobic conditions.
\end{abstract}

Keywords: Gluconate; Ethanol; Escherichia coli; Fermentation; Medium

\section{Introduction}

Cellulosic biomass is an attractive, renewable, abundant, and lowcost feedstock for fuel and chemical production [1]. Their biochemical conversion features cellulase mediated hydrolysis and microbial fermentation. The cellulosic biomass hydrolysate mainly contains hexose and pentose sugars, which can be fermented to various fermentation products including ethanol. Recently, copper-dependent lytic polysaccharide monooxygenases (LPMOs) were found to be able to catalyze the oxidative cleavage of cellulose, generating oxidized cellodextrins in concert with cellobiose dehydrogenases [2]. The addition of LPMOs to cellulase enzyme cocktails to enhance cellulose degradation generates cellobionic acid and gluconic acid in addition to pentose and hexose sugars in the hydrolysate [3]. Our lab proposed an alternative route for biofuel and chemical production from cellulosic biomass in which cellobionate instead of monomeric sugars is produced as the reactive intermediate for subsequent fermentation [4] Conversion of gluconic acid or cellobionic acid to biofuels is of importance for complete utilization of all the carbon sources available in biomass.

Both of the hydrolysis products from cellobionate, glucose and gluconate, were found to be utilized efficiently by E. coli KO11 [4]. From 1 mole of gluconate, 1.5 moles of ethanol and 0.5 moles of acetate were produced, while 2 moles of ethanol were produced from 1 mole of glucose. The rate of gluconate utilization by $E$. coli KO11 was even faster than that of glucose in Luria Broth (LB) medium [4]. Glucose and gluconate were utilized by E. coli KO11 simultaneously when both of them were present as the carbon source, and again gluconate was used faster than glucose. Although $E$. coli KO11 was an excellent ethanologen for ethanol production from glucose, and close to theoretical yield was achieved from glucose in LB medium, the ethanol yield from gluconate produced by this strain was about only $87 \%$ of the theoretical maximum [4,5]. E. coli KO11 was then engineered for improved ethanol production from gluconate. Deletion of the gene encoding pyruvate formate lyase (pfl) alone led to lower acetate yield, lower ethanol yield and more carbon flow to lactic acid production. Subsequent deletion of the L-lactate dehydrogenase (ldh) gene in addition to pfl eliminated lactate production, reduced the carbon flow toward acetate production, and improved the ethanol yield from $87.5 \%$ to $97.5 \%$ of the theoretical maximum. The resulting strain was named E. coli AH003 [6].

All previous work with E. coli AH003 has used complex growth media containing laboratory nutrients such as yeast extract and tryptone. The use of these nutrients for the industrial production of ethanol may not be feasible because of their high cost. In this study, we focus on the fermentation of glucose and gluconate to ethanol using a minimal mineral salts medium. Parent strain E. coli KO11 was used as a comparison.

\section{Materials and Methods}

\section{Bacterial strains and media}

Strains E. coli $\mathrm{KO} 11$ and E. coli $\mathrm{AH} 003$ were stocked at $-80^{\circ} \mathrm{C}$. Before each fermentation study, each strain was streaked onto a LB plate and incubated at $37^{\circ} \mathrm{C}$ overnight. Cells from a single colony were inoculated into a $15 \mathrm{~mL}$ centrifuge tube containing $8 \mathrm{~mL} \mathrm{LB}$. The culture was incubated at $37^{\circ} \mathrm{C}$ for 6 hours. $1 \mathrm{~mL}$ of the pre-culture was transferred to a $200 \mathrm{~mL}$ seed serum bottle containing $100 \mathrm{~mL}$ of $\mathrm{LB}$ 
with $2 \%$ glucose. The seed culture was incubated for 14 hours at $37^{\circ} \mathrm{C}$ and shaken at $200 \mathrm{rpm}$. Cells were harvested by centrifugation and used to inoculate fermentation serum bottles, which contained $100 \mathrm{~mL}$ of medium with various carbon sources, to a starting OD600 of 0.05 .

Two media were used in this study. They are LB medium and M9 medium. LB medium contained the following components: $20 \mathrm{~g} / \mathrm{L}$ tryptone, $10 \mathrm{~g} / \mathrm{L}$ yeast extract, and $10 \mathrm{~g} / \mathrm{L}$ sodium chloride. $\mathrm{M} 9$ medium contained $6 \mathrm{~g} / \mathrm{L} \mathrm{Na}_{2} \mathrm{HPO}_{4}, 3 \mathrm{~g} / \mathrm{L} \mathrm{KH}_{2} \mathrm{PO}_{4}, 1 \mathrm{~g} / \mathrm{L} \mathrm{NH} \mathrm{NH}_{4} \mathrm{Cl}, 0.5$ $\mathrm{g} / \mathrm{L} \mathrm{NaCl}, 0.002 \mathrm{M} \mathrm{MgSO}_{4} .7 \mathrm{H}_{2} \mathrm{O}, 0.0001 \mathrm{M} \mathrm{CaCl}_{2}$, and $1 \mathrm{mg} / \mathrm{L}$ thiamine-HCl. Glucose and potassium gluconate were added at various starting concentrations as the carbon source.

\section{Anaerobic fermentation and growth conditions}

The serum bottles were $\mathrm{pH}$ adjusted to 6.5 with hydrochloric acid. Bottles were purged with argon to obtain anaerobic conditions. All fermentations were run in a minimum of triplicates on a rotary shaker at $37^{\circ} \mathrm{C}$ and $200 \mathrm{rpm}$ using $200 \mathrm{~mL}$ serum bottles containing $100 \mathrm{~mL}$ of fermentation broth. Samples were taken out at various time intervals. The cell density was measured at $600 \mathrm{~nm}$ using a spectrophotometer. The concentrations of sugars, sugar aldonates, and fermentation products were analyzed using HPLC.

\section{Analytical procedures}

The concentrations of glucose, gluconate, acetic acid, and ethanol were measured by a Shimadzu HPLC equipped with a refraction index detector, PDA detector, and a Transgenomic ICSep ION-300 column (Transgenomic, San Jose, CA, USA) at $80^{\circ} \mathrm{C}$. Five millimolar sulfuric acid at a flow rate of $0.6 \mathrm{~mL} / \mathrm{min}$ was used as mobile phase. To measure the glucose and gluconate concentration in the glucose and gluconate co-fermentation experiments, the supernatant of the fermentation broth was analyzed using a Transgenomic CARBOSep COREGEL-87C (Transgenomic, San Jose, CA, USA) column at $80^{\circ}$ C. $20 \mathrm{mM}$ calcium chloride at a flow rate of $0.6 \mathrm{ml} / \mathrm{min}$ was used as the mobile phase.

\section{Results}

\section{Co-fermentation of glucose and gluconate in LB medium}

Figure 1 shows the fermentation of $50 \mathrm{mM}$ of glucose, gluconate and the mixture of gluconate and glucose to ethanol and acetate in the LB medium. AH003 completely utilized all $50 \mathrm{mM}$ gluconate in 5.5 hours, while it took 8.5 hours to completely consume $50 \mathrm{mM}$ of glucose. When both $50 \mathrm{mM}$ of gluconate and $50 \mathrm{mM}$ of glucose were co-fermented, it took about the same 5.5 hours to deplete all the gluconate and 8.5 hours to deplete all the glucose. The rates of glucose and gluconate utilization in the mixture were almost the same as when the other substrate was not present. The yield of ethanol from glucose was about $100 \%$, the yield of ethanol from gluconate was about $97 \%$, the ethanol yield from glucose and gluconate mixture was about $97 \%$.
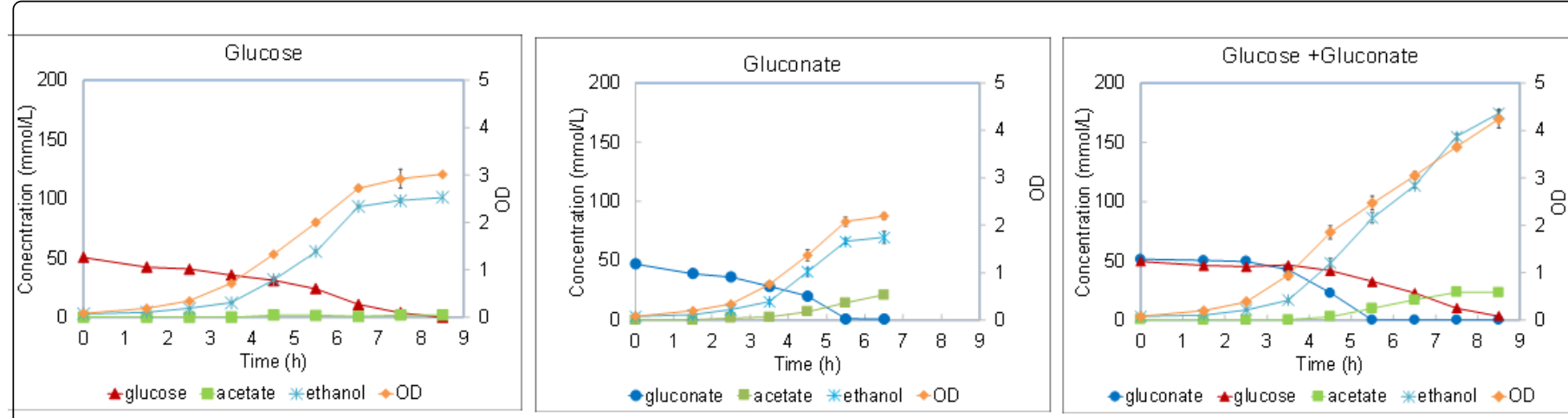

Figure 1: Time course of substrate utilization and product formation by E. coli AH003 strain in LB medium a) glucose, b) gluconate, c) glucose and gluconate. Error bars indicate standard deviations of the three repeats.

\section{Fermentation of glucose, gluconate and the glucose and gluconate mixture in the mineral salts medium}

The conversion of glucose, potassium gluconate and the mixture of glucose and potassium gluconate were evaluated using M9 mineral medium without any casamino acid addition. As shown in Figure 2, M9 mineral salts medium supported the growth of AH003 on gluconate. All gluconate was completely consumed within 17 hours, and the OD of the strain reached 1.0. About $62 \mathrm{mM}$ of ethanol and 16 $\mathrm{mM}$ of acetate were produced from $50 \mathrm{mM}$ of the gluconate. Ethanol yield was about $82 \%$ of the theoretical maximum. When glucose was used as the carbon source, the strain grew very slowly. The optical density of the strain was only about 0.2 , and only one third of the glucose was consumed after 32 hours. The ethanol yield from the consumed glucose was about $80 \%$ of the theoretical maximum. Interestingly, the strain used glucose much faster in the $50 \mathrm{mM}$ glucose and $50 \mathrm{mM}$ gluconate mixture than when glucose was present alone. All glucose was used in 30 hours, while all gluconate was used in 27 hours. The optical density of the culture reached 1.6. About $139 \mathrm{mM}$ ethanol was produced from the mixture, yielding $80 \%$ of the maximal theoretical yield. In the fermentation using AH003, no obvious succinate, lactate nor formate were detected in the fermentation broth, which aligned with the fact that the genes responsible for generating them such as frd, ldh and pfl were knocked out in the E. coli AH003 genome. 

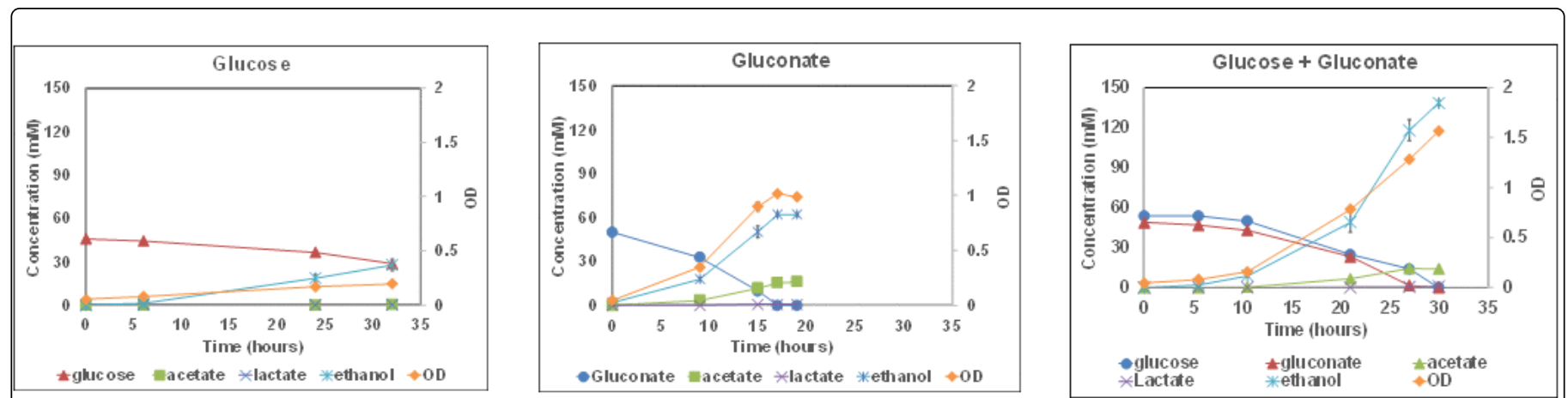

Figure 2: Time course of substrate utilization and product formation by E. coli AH003 strain in M9 medium a) glucose, b) gluconate; and c) glucose and gluconate. Error bars indicate standard deviations of the three repeats.
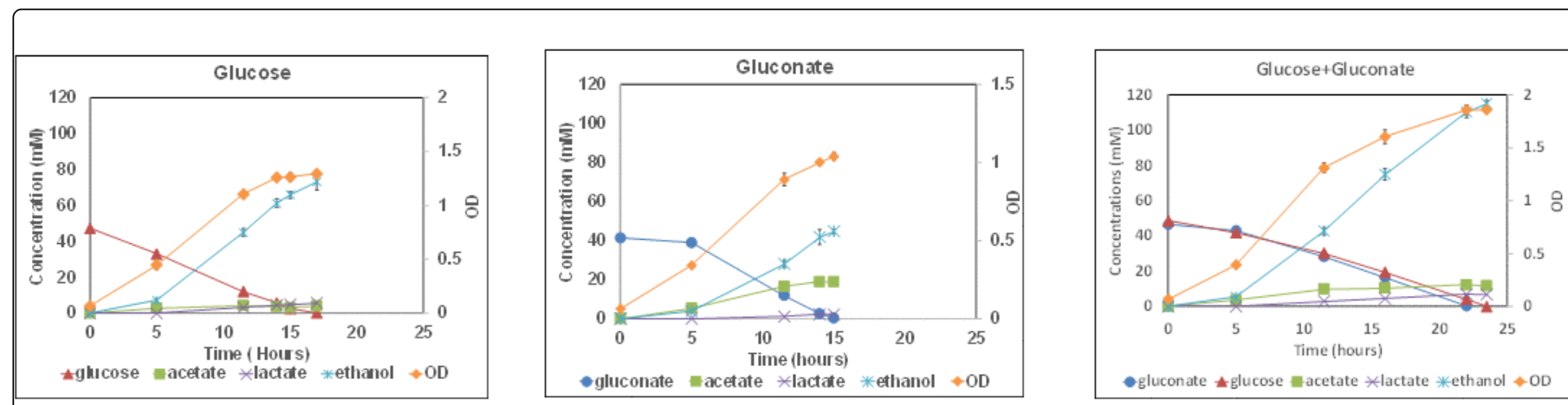

Figure 3: Time course of substrate utilization and product formation by E. coli KO11 strain in M9 medium a) glucose, b) gluconate; and c) glucose and gluconate. Error bars indicate standard deviations of the three repeats.

When E. coli KO11 was used as the ethanologen in M9 medium, we found that glucose was utilized not much slower than gluconate as shown in Figure 3. All glucose was used up in 16 hours, while all gluconate was used up in about 15 hours. Again, in the glucose and gluconate mixture, gluconate was used up in 22 hours, while glucose got used up in 23.5 hours. The addition of gluconate to glucose did not increase the rate of glucose utilization. The yields of ethanol from glucose, gluconate and their mixture were all about $75 \%$ of theoretical maximum. About $25-30 \mathrm{mM}$ formate and about $5 \mathrm{mM}$ lactate was produced in the fermentation broth. The rates of substrate utilization in all cases were faster than those of AH003.

\section{Discussion}

E. coli $\mathrm{KO} 11$ is one of the best ethanologens available to convert all biomass sugars such as glucose, xylose and arabinose to ethanol [7]. It contains chromosomally integrated genes encoding pyruvate decarboxylase (pdc) and alcohol dehydrogenase (adhB) from the Zymomonas mobilis PET operon [5]. The fumarate reductase (frd) gene was deleted to prevent succinate production and further improve the carbon flow toward ethanol production [5]. E. coli strains have distinct pathways to transport glucose and gluconate [8-11]. Gluconate does not seem to have any obvious effect on carbon catabolite repression of glucose and vice versa. The utilization of glucose and gluconate happens simultaneously. At low concentrations, the rates of glucose and gluconate utilization in co-fermentation were the same as when the other substrate was not present. When starting concentrations of glucose and gluconate in the co-fermentation were higher $(100 \mathrm{mM})$, the substrate utilization rate seemed to be slowed by one another, perhaps due to higher concentrations of inhibitory metabolites produced from higher starting substrate concentrations [4].

The deletion of $\mathrm{pfl}$ and ldh in E. coli KO11 yielded the strain E. coli AH003 [6]. Although the deletion of pfl and ldh did not have any obvious effect on ethanol yield from glucose in LB medium [6], it significantly affected the strain's growth on glucose in minimal salts medium. E. coli AH003 can barely grow on glucose. However, both strain $\mathrm{AH} 003$ and KO11 maintained good growth when gluconate was used as the substrate. In contrast to the poor growth on glucose in M9 medium, E. coli AH003 utilized glucose much faster when both glucose and gluconate were present. When KO11 was used as the ethanologen, the presence of gluconate did not improve the rate of glucose utilization in the glucose and gluconate co-fermentation.

These results can be explained by the absence of the $p f l$ gene in the AH003 genome. PFL and pyruvate dehydrogenase (PDH) are both responsible for acetyl-CoA production in E. coli. $\mathrm{PDH}$ is an enzyme that is strongly inhibited by $\mathrm{NADH}$ and requires $\mathrm{NAD}+$ for the activity [12]. It has minimal functionality under anaerobic conditions when the ratio of $\mathrm{NADH}$ to NAD+ is high [12], as PFL is the main route for acetyl-CoA production under anaerobic conditions. However, recent studies have shown that there is still flux through the PDH pathway for certain strains of $E$. coli under anaerobic conditions, which serves to maintain redox balance and provide reducing equivalents when 
glucose is the carbon source [13]. In E. coli AH003, the pfl gene was deleted. Thus, the PDH route is the only way for the cell to generate acetyl-CoA, which is the precursor for anabolism. When glucose, which is a much reduced substrate, was used, a high NADH/NAD+ ratio was generated which led to strong inhibition of the $\mathrm{PDH}$ and slower generation of acetyl-CoA. Consequently, AH003 grew on and fermented glucose very slowly. However, when gluconate was used as the substrate, a lower NADH/NAD+ level was achieved inside the cell since it is a more oxidized substrate [14]. As a result, PDH was less inhibited and normal growth and reasonable rate of gluconate utilization was achieved. When both glucose and gluconate were present in the co-fermentation, they were utilized simultaneously. The ratio of NADH/NAD+ should fall between the levels when glucose or gluconate were present alone. Hence, the gluconate utilization rate in the mixture was slower than that of gluconate alone, whereas the rate of glucose utilization was faster than that of glucose alone.

E. coli KO11 has an active PFL pathway. The formation of large amount of formate suggested that the $p f l$ gene was active and the acetyl-CoA was generated through the PFL pathway instead of the $\mathrm{PDH}$ pathway. Since the ratio of NADH/NAD+ did not significantly affect PFL, nor acetyl-CoA production and anabolism, the fermentation of glucose occurred not much slower than gluconate. The effect of gluconate enhancing the rate of glucose utilization was not seen in the glucose and gluconate co-fermentation in E. coli KO11. However, the ethanol yields achieved using E. coli KO11 were lower than those of E. coli AH003 due to the formation of other metabolites.

Productivity and nutrient costs are both significant factors in process economics for the microbial production of commodity products. In this sense, gluconate is a very desirable and unique substrate. In all media tested, gluconate was utilized faster than glucose. Besides, gluconate needs less rich supplemental nutrients than glucose to support E. coli AH003's growth and product formation. The addition of gluconate could improve the glucose utilization rate in cofermentation in minimal mineral salts medium. The deletion of the $\mathrm{pfl}$ gene to redirect carbon flow toward the target products is a very common strategy used in strain engineering for biofuels and chemicals production. We expect that what we observed with E. coli AH003 is likely applicable to strains with similar features too. Strains with $p f l$ deleted normally need relatively rich media to support anaerobic growth and product formation [15,16]. The co-fermentation of gluconate and glucose could be a useful strategy to improve the rate of glucose fermentation and decrease nutrient requirements for engineered strains lacking the $p f l$ gene grown under anaerobic conditions.

\section{Acknowledgements}

This project was supported by Agriculture and Food Research Initiative Competitive Grant No. 2011-67009-20060 from the USDA National Institute of Food and Agriculture. The financial support to Yaqin Sun and Shuangyan Han from China Scholarship Council and editorial help from Eric Walters and Amanda Hildebrand are gratefully acknowledged.

\section{References}

1. Zaldivar J, Nielsen J, Olsson L (2001) Fuel ethanol production from lignocellulose: a challenge for metabolic engineering and process integration. Appl Microbiol Biotechnol 56: 17-34.

2. Beeson WT, Phillips CM, Cate JHD, Marletta MA (2012) Oxidative Cleavage of Cellulose by Fungal Copper-Dependent Polysaccharide Monooxygenases. J Am Chem Soc 134: 890-892.

3. Li X, Chomvong K, Yu VY, Liang JM, Lin YP, Cate, et al. (2015) Cellobionic acid utilization: from Neurospora crassa to Saccharomyces cerevisiae. Biotechnology for biofuels 8: 120 .

4. Fan Z, Wu W, Hildebrand A, Kasuga T, Zhang R, et al.(2012) A novel biochemical route for fuels and chemicals production from cellulosic biomass. PloS one 7: e31693.

5. Ohta K, Beall DS, Mejia JP, Shanmugam KT, Ingram LO (1991) Genetic improvement of Escherichia coli for ethanol production: chromosomal integration of Zymomonas mobilis genes encoding pyruvate decarboxylase and alcohol dehydrogenase II. Appl Environ Microbiol 57: 893-900.

6. Hildebrand A, Schlacta T, Warmack R, Kasuga T, Fan Z (2013) Engineering Escherichia coli for improved ethanol production from gluconate. J Biotechnol 168: 101-106.

7. Ingram LO, Aldrich HC, Borges AC, Causey TB, Martinez A, et al. (1999) Enteric bacterial catalysts for fuel ethanol production. Biotechnol Prog 15: 855-866.

8. Ponce E, Garcia M, Munoz ME (2005) Participation of the EntnerDoudoroff pathway in Escherichia coli strains with an inactive phosphotransferase system (PTS- Glc+) in gluconate and glucose batch cultures. Can J Microbiol 51: 975-982.

9. Gomez KM, Rodriguez A, Rodriguez Y, Ramirez AH, Isturiz T (2011) The subsidiary GntII system for gluconate metabolism in Escherichia coli: Alternative induction of the gntV gene. Biol Res 44: 269-275.

10. Porco A, Peekhaus N, Bausch C, Tong S, Isturiz T, et al. (1997) Molecular genetic characterization of the Escherichia coli gntT gene of GntI, the main system for gluconate metabolism. J Bacteriol 179: 1584-1590.

11. Tong S, Porco A, Isturiz T, Conway T (1996) Cloning and molecular genetic characterization of the Escherichia coli gntR, gntK, and gntU genes of GntI, the main system for gluconate metabolism. J Bacteriol 178: 3260-3269.

12. Wang Q, Ou MS, Kim Y, Ingram LO, Shanmugam KT (2010) Metabolic flux control at the pyruvate node in an anaerobic Escherichia coli strain with an active pyruvate dehydrogenase. Appl Environ Microbiol 76: 2107-2114.

13. Murarka A, Clomburg JM, Moran S, Shanks JV, Gonzalez R (2010) Metabolic analysis of wild-type Escherichia coli and a pyruvate dehydrogenase complex (PDHC)-deficient derivative reveals the role of PDHC in the fermentative metabolism of glucose. J Boil Chem 285: 31548-31558.

14. Sanchez AM, Bennett GN, San KY (2005) Effect of different levels of NADH availability on metabolic fluxes of Escherichia coli chemostat cultures in defined medium. J Biotechnol 117: 395-405.

15. Atsumi S, Cann AF, Connor MR, Shen CR, Smith KM, et al. (2008) Metabolic engineering of Escherichia coli for 1-butanol production. Metab Eng 10: 305-311.

16. Atsumi S, Hanai T, Liao JC (2008) Non-fermentative pathways for synthesis of branched-chain higher alcohols as biofuels. Nature 451: 86-89. 